\title{
The WRKY transcription factor PIWRKY65 enhances the resistance of Paeonia lactiflora (herbaceous peony) to Alternaria tenuissima
}

\author{
Xue Wang ${ }^{1}$, Junjie $\mathrm{Li}^{1}$, Jing Guo ${ }^{1,2}$, Qian Qiao ${ }^{3}$, Xianfeng Guo ${ }^{1,2}$ and Yan Ma ${ }^{1,2}$
}

\begin{abstract}
In this study, the disease resistance gene PIWRKY65 was isolated from the leaves of Paeonia lactiflora and analyzed by bioinformatics methods, and the localization of the encoded protein was explored. Quantitative real-time PCR (qRT$\mathrm{PCR}$ ) was also used to explore the response of this gene to Alternaria tenuissima. The results showed that the gene sequence contained multiple cis-acting elements involved in the response to hormone signaling molecules belonging to the lle subgroup of the WRKY family, and the encoded proteins were located in the nucleus. The PIWRKY65 gene has a positive regulatory effect on A. tenuissima infection. After silencing the PIWRKY 65 gene via virus-induced gene silencing (VIGS), it was found that the gene-silenced plants were more sensitive to $A$. tenuissima infection than the wild plants, exhibiting more severe infection symptoms and different degrees of changes in the expression of the pathogenesis-related (PR) genes. In addition, we showed that the endogenous jasmonic acid (JA) content of $P$. lactiflora was increased in response to A. tenuissima infection, whereas the salicylic acid (SA) content decreased. After PIWRKY65 gene silencing, the levels of the two hormones changed accordingly, indicating that PIWRKY65, acting as a disease resistance-related transcriptional activator, exerts a regulatory effect on JA and SA signals. This study lays the foundation for functional research on WRKY genes in P. lactiflora and for the discovery of candidate disease resistance genes.
\end{abstract}

\section{Introduction}

Plant immunity is a complex, multilayered system that includes several defense mechanisms. In addition to passive defense mechanisms, such as the cuticle, cell wall, and antimicrobial compounds, plants exhibit two layers of active defense mechanisms. The first is pattern-triggered immunity (PTI), induced by pathogen-associated molecular patterns (PAMPs), and the second is effect-triggered immunity (ETI), induced by pathogen proteins ${ }^{1}$. When an immune response occurs in plants, the expression of

Correspondence: Xianfeng Guo (guoxf@sdau.edu.cn) or Yan Ma (myiris@sina.com)

${ }^{1}$ College of Forestry, Shandong Agricultural University, No. 61, Daizong Road, 271018 Tai'an, Shandong, China

${ }^{2}$ Shandong Provincial Research Center of Demonstration Engineering

Technology for Urban and Rural Landscapes, 271018 Tai'an, Shandong, China Full list of author information is available at the end of the article

These authors contributed equally: Xue Wang, Junjie Li disease resistance genes in cells is regulated by transcription factors (TFs), including the WRKYs, which constitute one of the largest transcription factor families and are involved in disease resistance ${ }^{2}$.

The WRKY TF family is named for the highly conserved WRKYGQK heptapeptide sequence in the $\mathrm{N}$-terminal amino acid sequence of its members ${ }^{3}$. Because of the presence of different numbers of WRKY domains and different zinc finger motifs, the WRKY TFs can be divided into three groups. Based on the phylogenetic tree of WRKY protein domains, group II can be divided into five subgroups $(\mathrm{IIa}-\mathrm{e})^{4}$. Studies have shown that when induced by external stimuli in plants, WRKY TFs are regulated by a cascade of defense signaling networks that bind with the promoters of downstream genes to regulate their expression and enhance plant defense ${ }^{5}$. Among plant disease resistance-related responses, the most

\section{(c) The Author(s) 2020}

(c) (i) Open Access This article is licensed under a Creative Commons Attribution 4.0 International License, which permits use, sharing, adaptation, distribution and reproduction cc) in any medium or format, as long as you give appropriate credit to the original author(s) and the source, provide a link to the Creative Commons license, and indicate if changes were made. The images or other third party material in this article are included in the article's Creative Commons license, unless indicated otherwise in a credit line to the material. If material is not included in the article's Creative Commons license and your intended use is not permitted by statutory regulation or exceeds the permitted use, you will need to obtain permission directly from the copyright holder. To view a copy of this license, visit http://creativecommons.org/licenses/by/4.0/. 
widely studied is the regulation of the salicylic acid (SA) and jasmonic acid (JA) signaling pathways. A great deal of scientific research has demonstrated that the WRKY family is directly or indirectly involved in these two signaling pathways ${ }^{6}$. For example, GbWRKY1 mediates the disease resistance and development of cotton through the JA signaling pathway and the negative regulation of cotton resistance to Botrytis cinerea ${ }^{7}$. Rice OsWRKY13 can directly or indirectly regulate the expression of upstream and downstream genes of JA and SA to inhibit JA synthesis-related and JA-responsive gene expression and activate SA synthesis-related and SA-responsive gene expression to participate in disease resistance in rice ${ }^{8}$.

Herbaceous peony is a common ornamental plant in China that has both economic and cultural value. Red spot is a serious disease of herbaceous peony caused by Alternaria alternata ( $A$. alternata) and Alternaria tenuissima (A. tenuissima $)^{9}$ that hinders peony production and causes serious economic loss. Moreover, the large-scale infection of peonies affects the growth and development of the underground bud, thereby affecting plant production in the second year. In recent years, the roles of WRKY TFs in disease resistance in plants have been widely reported. In Solanum pimpinellifolium, SpWRKY1, SpWRKY3, and SpWRKY6 participate in resistance against Phytophthora infestans as positive regulators ${ }^{10-12}$. Overexpression of OsWRKY13 may improve the ability of rice to resist bacterial blight, which is a common problem in rice ${ }^{8}$. However, no studies on the response of the Paeonia lactiflora WRKY family to pathogen stress have been reported. In this research, we identified a differentially expressed gene, PlWRKY65, from transcriptome data collected under infection with $A$. tenuissima. Studies have shown that PlWRKY65 can positively regulate the resistance of herbaceous peony to $A$. tenuissima and may play a role through either direct or indirect involvement in SA- and JA-mediated disease resistance signaling pathways.

\section{Results}

\section{Cloning and sequence analysis of PIWRKY65}

The genomic DNA and cDNA of P. lactiflora 'Da Fugui' were used as templates for amplification, and sequences with lengths of 1044 and $825 \mathrm{bp}$ were obtained, respectively. The corresponding GenBank accession number is KY271096. The obtained gene was named PlWRKY65 due to its high homology with Arabidopsis AtWRKY65 (Fig. 1a). By comparing genomic DNA with cDNA sequences, PlWRKY65 was found to contain three exons and two introns (Fig. 1b), and the second intron was a typical Rtype intron in the conserved WRKY domain.

Furthermore, PlantCARE software was used to analyze the PlWRKY65 gene sequence, and the results confirmed that the intron sequences of PlWRKY65 contain 14 CAAT box components, 6 TATA box components, G-box light- responsive components, several hormone signaling molecule-responsive cis-elements, such as methyl jasmonic acid (MeJA) signal elements, and cis-elements involved in the metabolic regulation of proteins.

A phylogenetic tree of PlWRKY65 and Arabidopsis WRKY family genes was constructed by referring to the standard classification of Arabidopsis WRKY members to carry out phylogenetic analysis and clustering ${ }^{3}$. The results showed that PIWRKY65 and AtWRKY65 formed a branch belonging to the IIe subgroup of the WRKY family (Fig. 1c). Multiple comparative analyses indicated that the PIWRKY65 gene-coding region contains a WRKY domain structure consisting of 58 (152-209) amino acids, and this structure shares high homology with other plant WRKY65 TFs. In the $\mathrm{N}$-terminal domain, the conserved structure contains the highly conserved WRKYGQK heptapeptide sequence, and the C-terminus harbors a $\mathrm{C}_{2} \mathrm{H}_{2}\left(\mathrm{CX}_{5} \mathrm{CX}_{23} \mathrm{HNH}\right)$ zinc finger-type structure (Fig. 1d). The phylogenetic tree results showed that among the analyzed WRKY65 proteins, P. lactiflora PIWRKY65 was the most closely related to Nelumbo nucifera NnWRKY65 (Fig. 1e).

\section{Subcellular localization of the PIWRKY65 protein}

We transformed the recombinant vector pROKIIPlWRKY65-GFP and the vector containing only GFP into tobacco leaf epidermal cells to study the specific sites of the PIWRKY65 protein in cells. The results showed that green fluorescence in the epidermal cells of tobacco leaves transformed with the recombinant vector was present only in the nucleus, while in the leaves of the control group, the fluorescence showed a diffuse distribution and was present in both the nucleus and cytoplasm. Thus, it was inferred that the PlWRKY65 protein is present in the nucleus and may play a role there (Fig. 2).

\section{PIWRKY65 was positively induced by $A$. tenuissima}

Based on the differential expression of PlWRKY65 according to the transcriptome data, we further studied the expression patterns of PlWRKY 65 after infection with A. tenuissima. The results showed that PlWRKY65 was positively expressed during infection with $A$. tenuissima. The expression of PlWRKY65 increased sharply and peaked at $24 \mathrm{~h}$ after infection with $A$. tenuissima, at a level 16.76 times higher than that of the control. Thereafter, the expression level remained higher than that of the control group until $96 \mathrm{~h}$, showing an overall upward trend (Fig. 3).

\section{VIGS of PIWRKY65 reduced the transcript abundance of endogenous PIWRKY65}

To preliminarily understand the mechanism of PlWRKY65 in response to infection with A. tenuissima, we further evaluated the functions of PlWRKY65 by silencing. Leaf cDNAs obtained after 10 days of infection with the empty vector TRV::00 and recombinant vector 
a

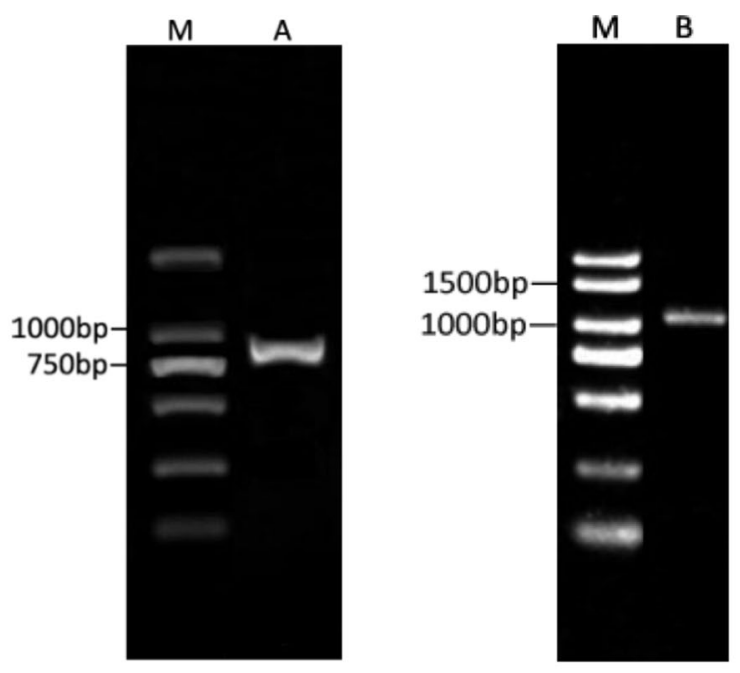

b

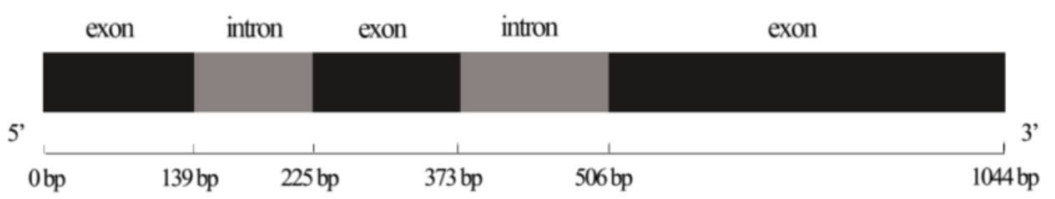

C

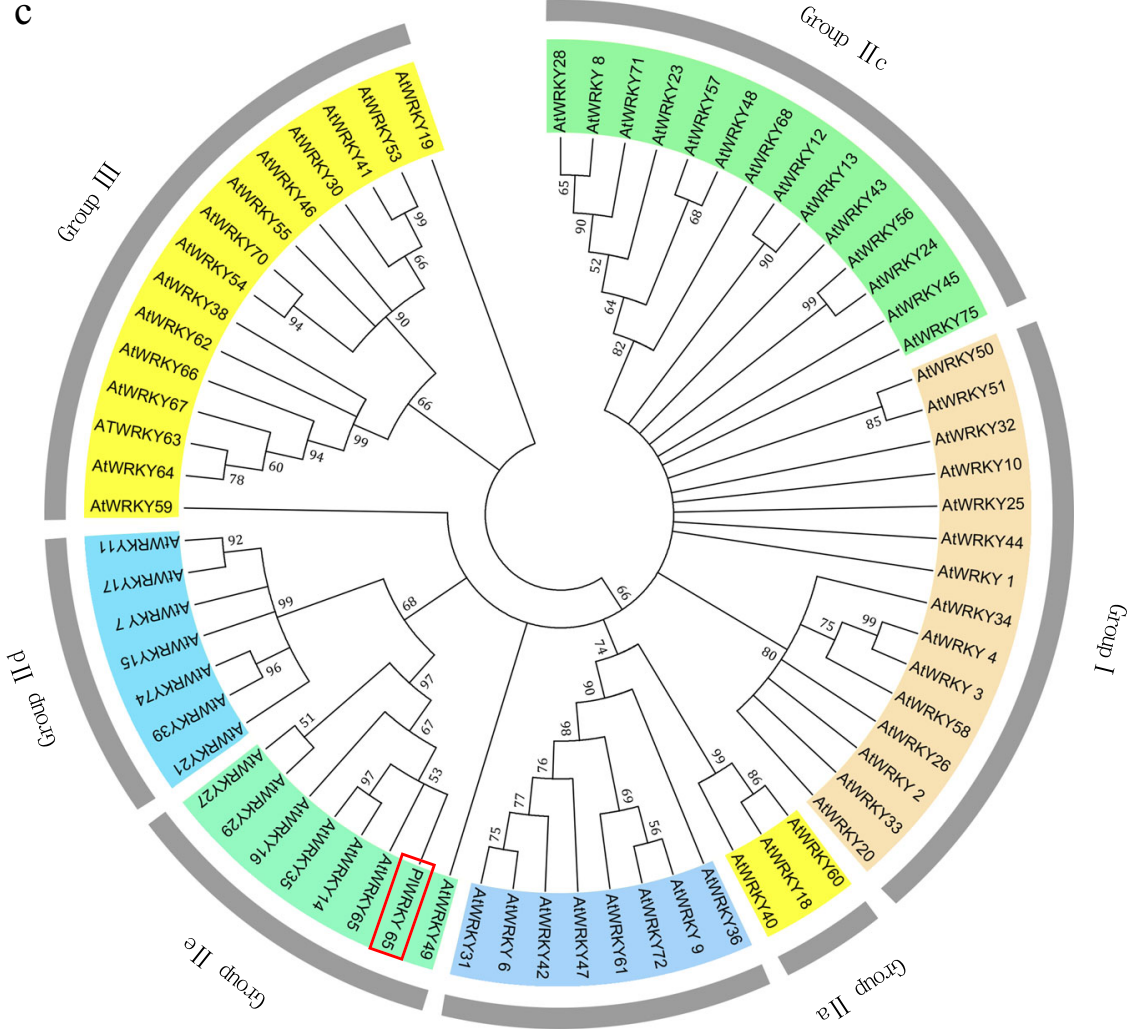

qII dnoxg 
$\mathrm{d}$

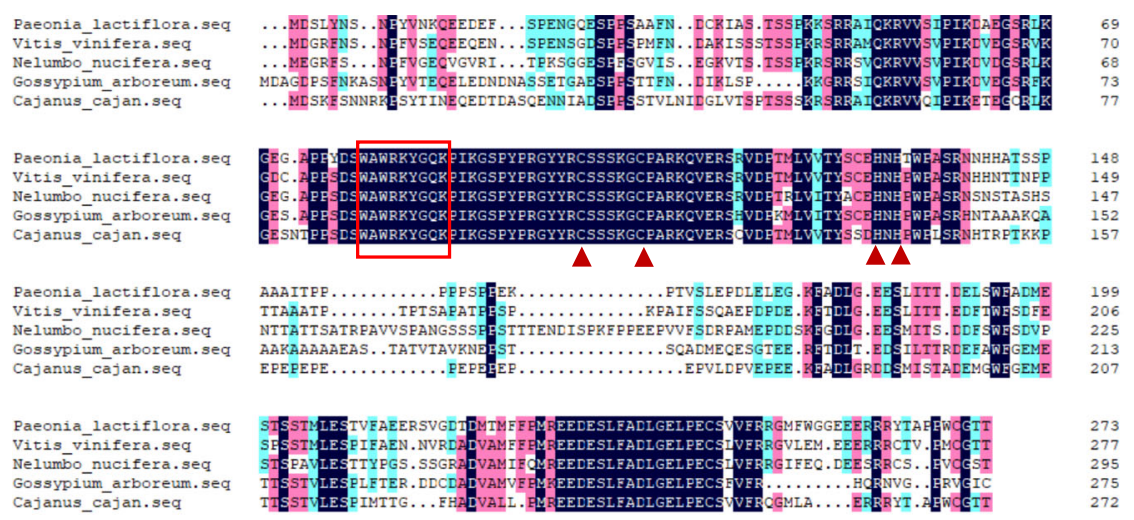

e
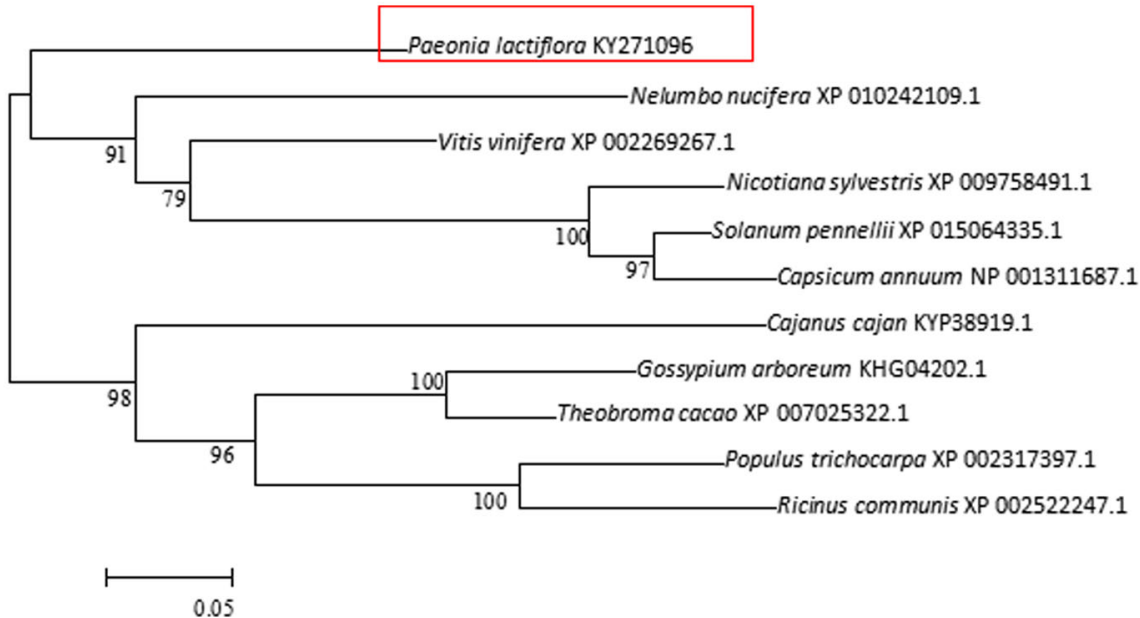

Fig. 1 Bioinformatics analysis of PIWRKY65. a PCR amplification products of pIWRKY65: M, DNA marker DL2000; A, PIWRKY65 cDNA fragment; B, PIWRKY65 genomic DNA fragment. b Structure of the PIWRKY65 sequence. $\mathbf{c}$ Phylogenetic analysis based on the amino acid sequences of PIWRKY65 and WRKY family genes in Arabidopsis. d Multiple alignment of the deduced PIWRKY65 amino acid sequences with its homologs. Note: The box indicates a WRKYGQK heptapeptide sequence; the triangle indicates the zinc finger structure. $\mathbf{e}$ The phylogenetic tree derived from the alignment of the amino acid sequences of PIWRKY65 and other WRKY65

TRV::WRKY65 were used as templates, and pTRV1-F/R and pTRV2-F/R were used for PCR-based detection (Fig. 4a). The results showed that the target bands of $647 \mathrm{bp}$ (pTRV1) and $372 \mathrm{bp}$ (pTRV2) could be amplified from the $P$. lactiflora samples inoculated with TRV::00, and bands of 647 bp (pTRV1) and 774 bp (pTRV2::WRKY65) could be amplified from the samples inoculated with TRV:: WRKY65 (Fig. 4b). These findings indicated that TRV::00 and TRV::WRKY65 were successfully inserted and expressed in the genome of $P$. lactiflora.

The silencing of the PlWRKY 65 gene in the leaves of $P$. lactiflora was detected by qRT-PCR. The leaves were treated with TRV::00 or TRV::WRKY65 (with blank control), and after 16 days of treatment, the leaves were collected, and RNA was extracted. The results showed that $P l W R K Y 65$ gene expression in the leaves infected with TRV::WRKY65 was markedly lower than that in the blank control and the leaves infected with TRV::00 (Fig. 4c). These findings indicated that PlWRKY65 was effectively silenced in TRV:: WRKY65-infected leaves.

\section{PIWRKY65-silenced plants exhibited greater sensitivity to}

\section{A. tenuissima}

The control plants and the plants silenced for 20 days (silencing efficiency $>60 \%$ ) were selected for the pathogen infection test. As shown in Fig. 5a, the expression of PlWRKY65 in response to infection with A. tenuissima in the PlWRKY65-silenced plants was markedly lower than that in the two control groups, but the changes in expression were consistent with those of the control plants. These findings indicated that the TRVPlWRKY65-silencing vector effectively inhibited the expression of the PlWRKY65 gene, and in the PlWRKY65silenced plants, PlWRKY65 still played an active regulatory role against $A$. tenuissima. 


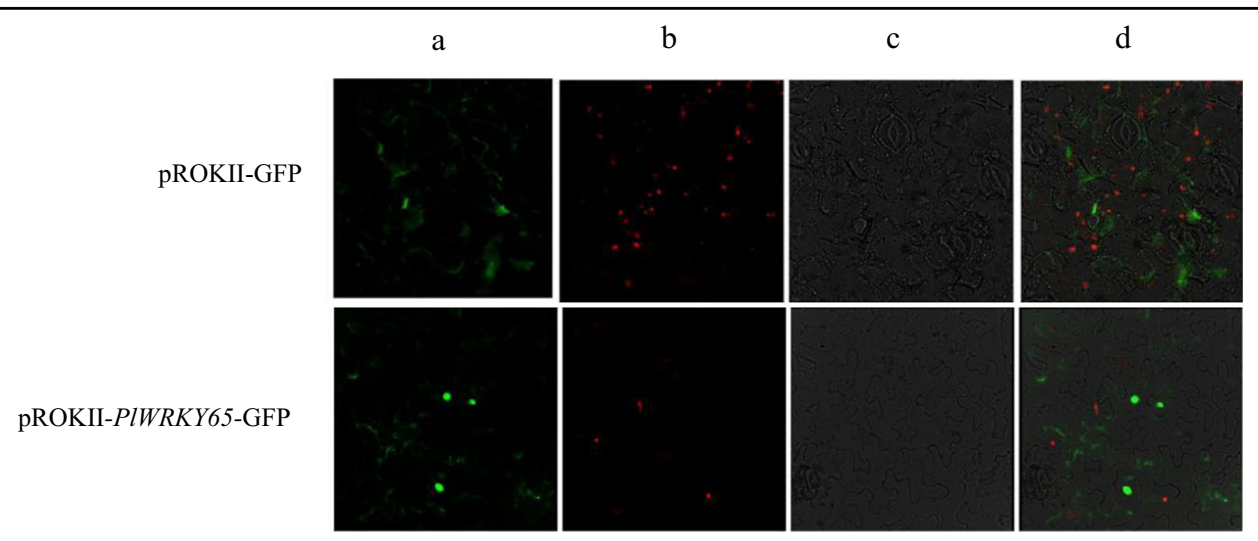

Fig. 2 Subcellular localization of the PIWRKY65 protein in Nicotiana benthamiana. a Fluorescent image, b chloroplast auto-fluorescence image, c natural light image, and $\mathbf{d}$ merged image

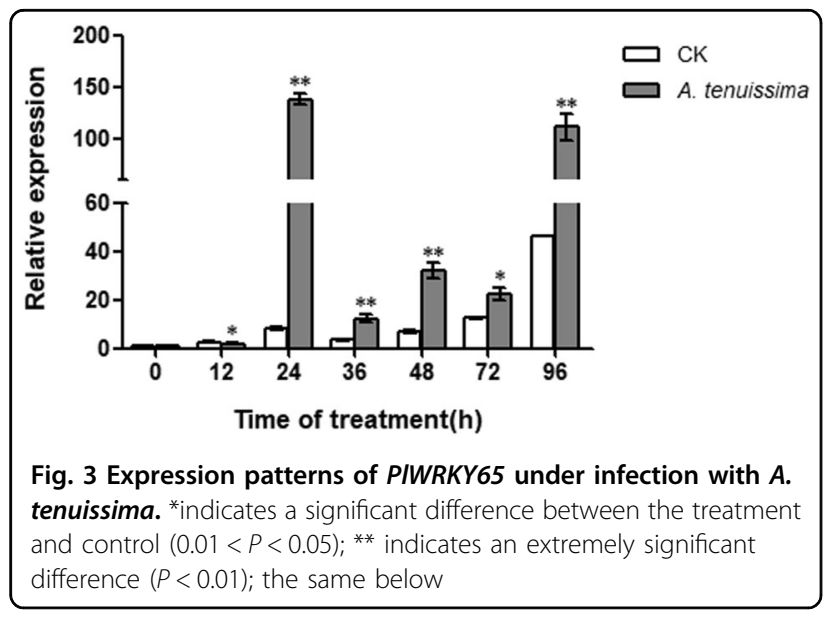

After infection with A. tenuissima, PlWRKY65-silenced plants exhibited higher sensitivity to the pathogens than the control plants (Fig. 5b), which was characterized by severe spreading of disease spot areas and parched perforation. After 21 days of infection, the incidence statistics of the plants showed that all plants were infected by $A$. tenuissima, and the disease indexes of the blank control plants, empty vector control plants, and PlWRKY65silenced plants were $43.05,44.10$, and 62.45 , respectively. The disease index of the PlWRKY65-silenced plants was markedly higher than that of the control (Fig. 5c) and was $19.40 \%$ and $18.35 \%$ higher than those of the blank control group and the empty vector control group, respectively. All of the above results illustrated that the PlWRKY65 gene was involved in the resistance of peony to $A$. tenuissima and played an active role in regulating disease resistance in peony by increasing its expression.

\section{PIWRKY65 expression participates in JA and SA signaling}

To investigate whether endogenous hormones respond to resistance to A. tenuissima and whether PlWRKY65 is associated with related hormones, we detected the endogenous levels of JA and SA in PlWRKY65-silenced plants and normal plants infected with A. tenuissima over $96 \mathrm{~h}$. The results revealed (Fig. 6a) that the JA content of the control plants increased overall from 0 to $96 \mathrm{~h}$, reaching the highest level at $96 \mathrm{~h}$. However, SA decreased from 0 to $48 \mathrm{~h}$, then increased to a peak at $72 \mathrm{~h}$ and decreased again thereafter. Interestingly, the JA level in the PlWRKY65silenced group was lower than that in the normal group and gradually recovered to the same level as that in the normal plants at $96 \mathrm{~h}$, but the SA level in the PlWRKY65silenced group was higher than that in the normal group and recovered to the same level as that in the normal plants at $96 \mathrm{~h}$, or even to a slightly lower level than that in the control. These data revealed that the changes in endogenous JA and SA levels are closely related to the disease resistance process after infection with $A$. tenuissima and are specifically correlated with the expression of PlWRKY65.

\section{Differential regulation of pathogenesis-related genes by PIWRKY65}

We conjectured that PlWRKY65 might be involved in the JA and SA signaling pathways because of the changes in endogenous hormones in PlWRKY65-silenced plants and the hormone signal-response elements in the PlWRKY65 gene. Therefore, we used qRT-PCR to analyze the expression of the JA and SA pathogenesis-related (PR) genes PR1, PR2, PR4B, PR5, and PR10 to verify this hypothesis. The results showed that only PIPR1 expression in PlWRKY65-silenced plants was markedly higher than that in control plants after $96 \mathrm{~h}$ of infection with $A$. tenuissima, while the expression of the other four PlPRs in PlWRKY65-silenced plants was markedly lower than that in the control plants (Fig. 6b). These results further indicated that PlWRKY65 regulated the resistance of $P$. lactiflora to A. tenuissima by participating in the JA and SA signaling pathways. 
a
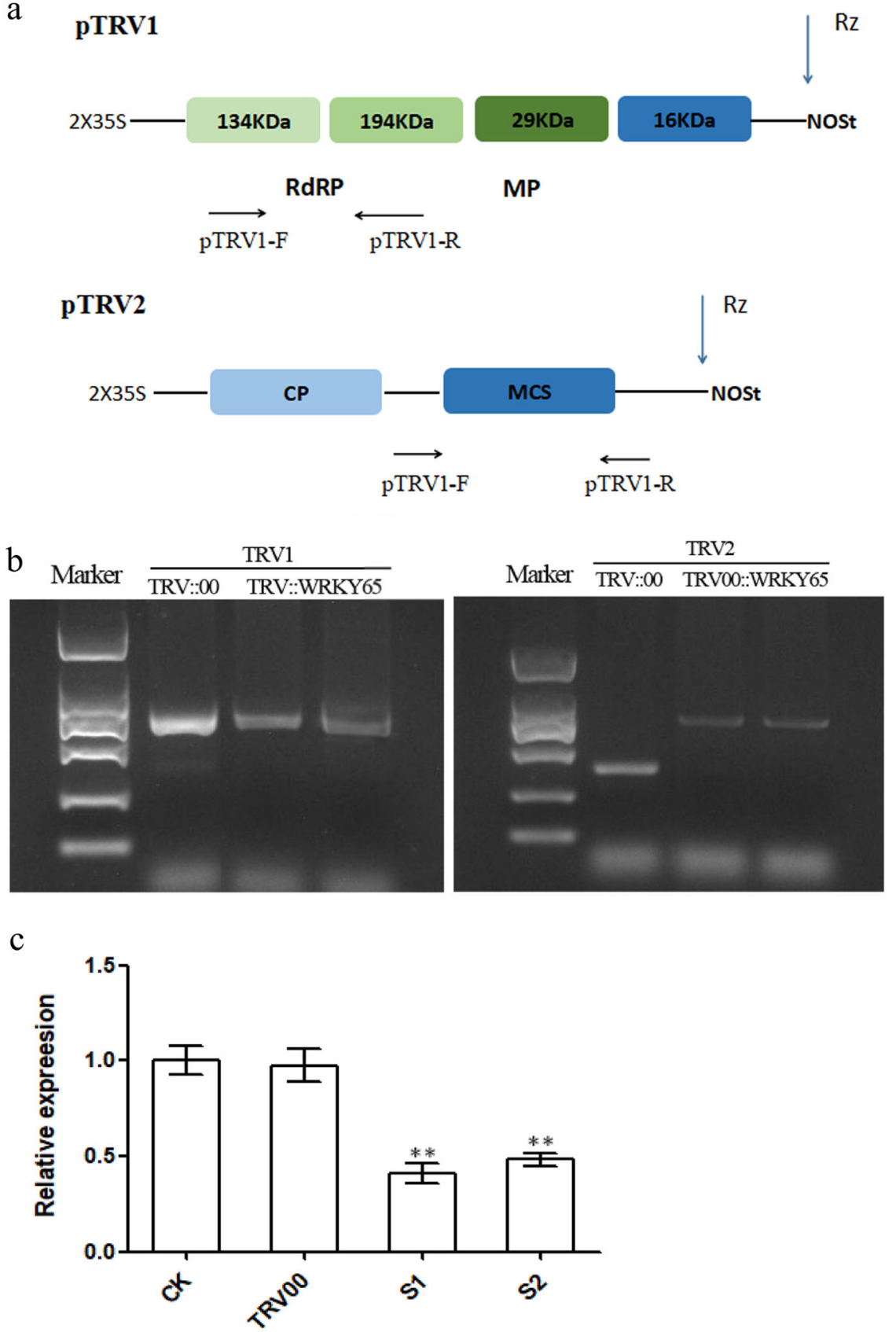

Fig. 4 The effect of PIWRKY65 silencing in VIGS plants. a Schematic diagram of the pTRV1 and pTRV2 plasmids and primers. $\mathbf{b}$ The PCR identification of RNA1 and RNA2 of TRV in P. lactiflora leaves. c PIWRKY65 gene expression levels in PIWRKY65-silenced plants. S1 and S2 are both TRV:: WRKY65 plants and are independent of each other

\section{Discussion}

The transcriptional regulation of defense genes plays indispensable roles in plant resistance. Therefore, the identification of the components of the plant defense system and corresponding response pathways is an important step for understanding plant stress resistance. The WRKY TF family, which is involved in disease responses, was identified recently, and many of its members are involved in the pathogen resistance response, as shown for the Arabidopsis thaliana WRKY7, $33,22,70$, and $54 \mathrm{TFs}$, which are directly involved in resistance to fungi ${ }^{13-16}$. However, most studies on these TFs have been conducted in A. thaliana, tomato, tobacco, potato, rice, cotton, and other plants ${ }^{17-21}$, and research on the gene functions of peony WRKY TFs is very limited. We screened a differentially expressed PlWRKY65 gene 
a

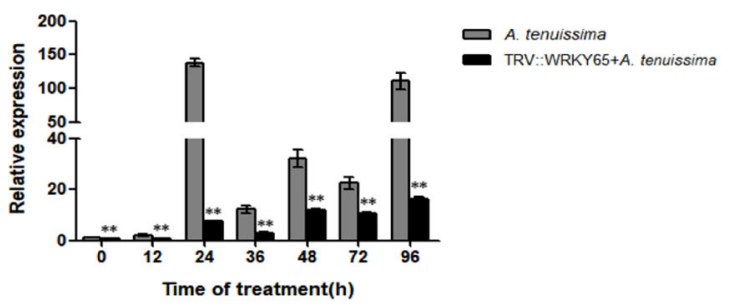

b

$7 \mathrm{~d}$

21d
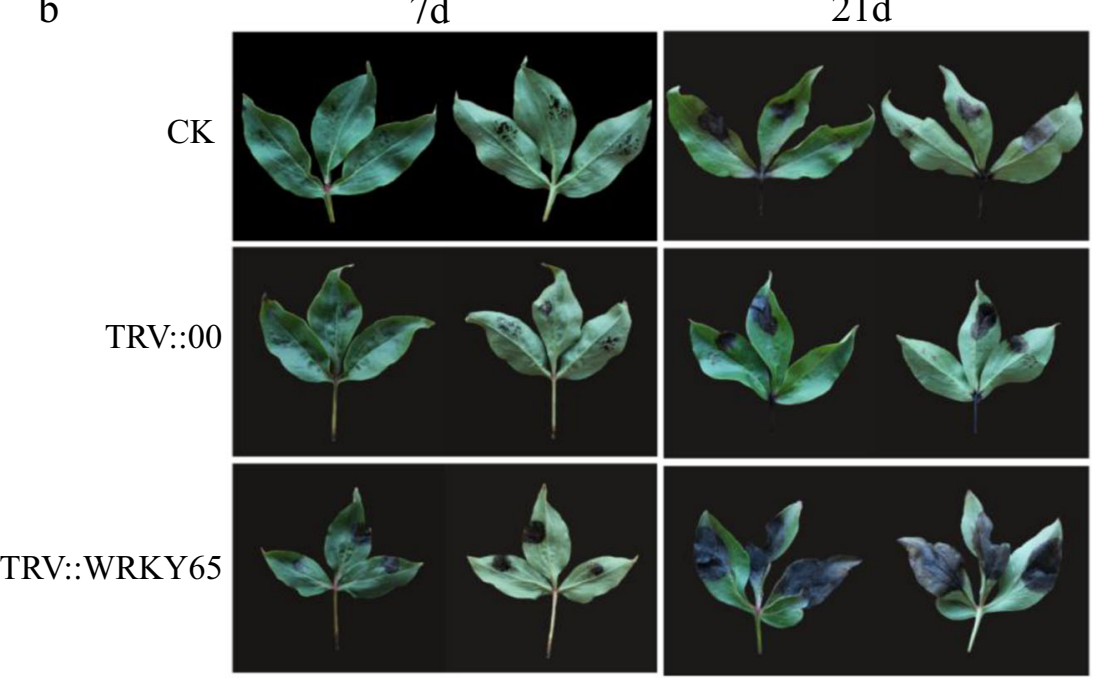

$\mathrm{c}$

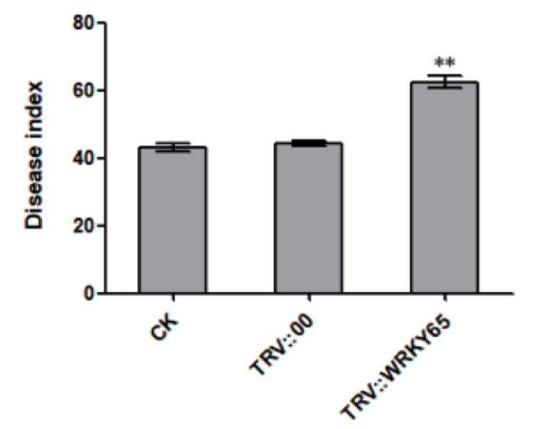

Fig. 5 Phenotypic differences in PIWRKY65-silenced plants in response to A. tenuissima. a Expression of PIWRKY65 in response to A. tenuissima before and after gene silencing. $\mathbf{b}$ Leaf phenotype under different treatments. $\mathbf{c}$ Disease index of plants treated with different treatments

from the transcriptome data of peony, speculated that it was related to the regulation of disease resistance, and provided strong evidence of the involvement of this gene in disease response regulation.

Regulatory elements involved in gene expression have been found in the introns of many genes. The introns of PlWRKY65 contain TATA box components, G-box lightresponsive components and several hormone signaling molecule-responsive cis-elements. These characteristics indicate that the introns of this gene may increase the transcription of the gene and participate in hormone regulation or synthesis and other plant developmental processes. Theoretically, we can establish a comparison between WRKY TFs from other plants and peony WRKYs and infer the potential functions of homologous WRKY proteins according to the known functions of these proteins. Previous studies have shown that the Arabidopsis AtWRKY65 gene is involved in the immune response related to $A t F L D^{22}$. Based on the fact that PlWRKY65 and AtWRKY65 both belong to the IIe subgroup, it is speculated that PlWRKY65 may be involved in the stress response during plant growth. The subcellular localization results implied that the PIWRKY65 protein was present in the nucleus, which agreed with the results obtained for 


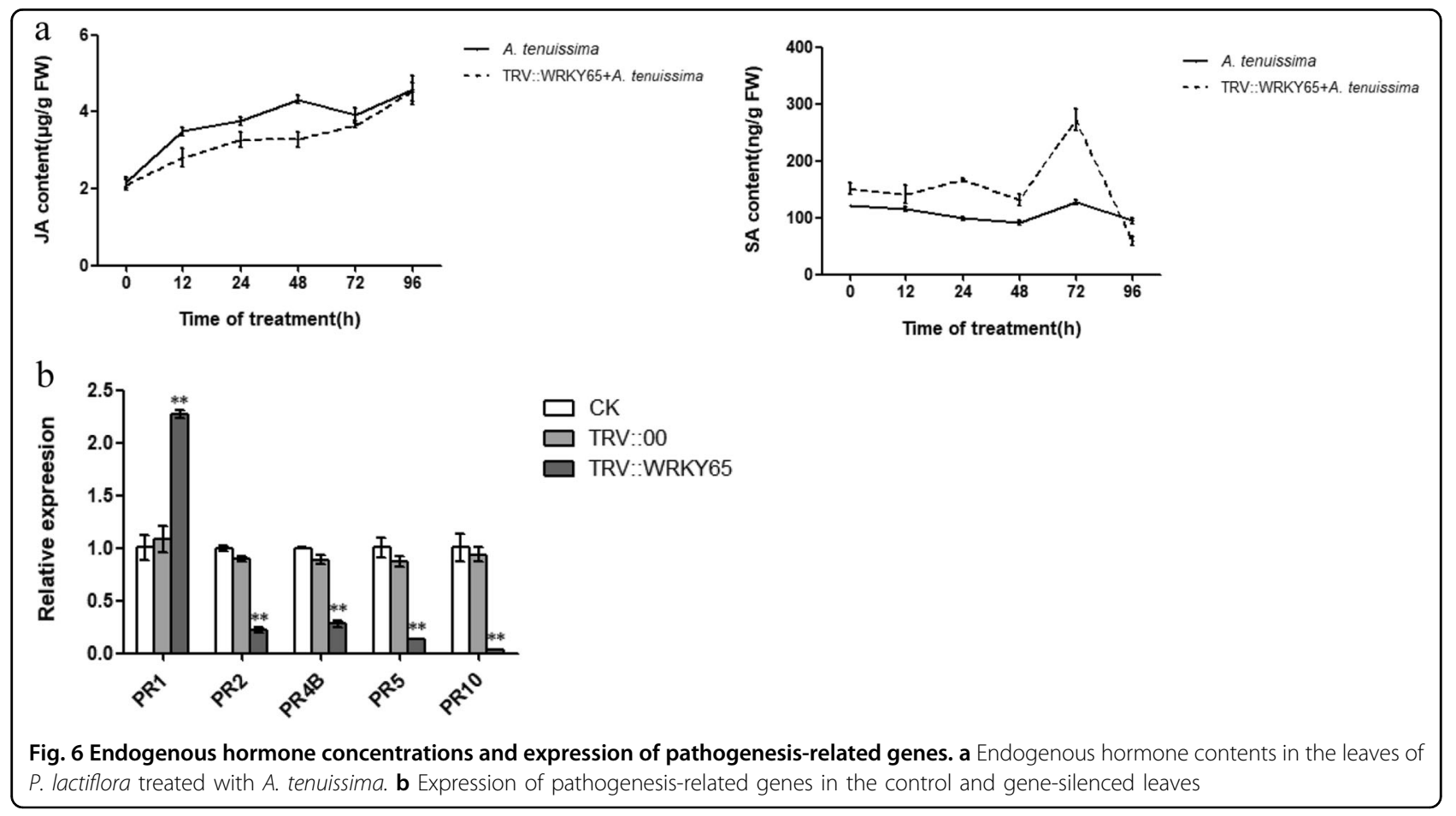

WRKY proteins in other species ${ }^{23-25}$. Therefore, we speculated that the PIWRKY65 protein acts as a transcriptional regulator, similar to most other WRKY proteins, and activates the expression of downstream target genes by binding to the $\mathrm{W}$-boxes of target genes.

TFs, especially those of the WRKY family, play an important role in plant disease resistance responses as signaling pathway regulators. AtWRKY8 and AtWRKY28, which belong to the IIa subgroup, can positively regulate the resistance of plants to $B$. cinerea ${ }^{26,27}$. Studies on the overexpression of OsWRKY45-1 and OsWRKY45-2 showed that this pair of alleles had a positive regulatory effect on resistance to Magnaporthe grisea, a fungal pathogen of rice ${ }^{6}$. The same conclusion was reached in this study, in which the expression of PlWRKY65 increased under induction by $A$. tenuissima and positively regulated the disease resistance of peony. However, in tomato, SlWRKY7O transcription negatively regulates plant resistance to fungi ${ }^{28}$. Therefore, WRKY TFs may be positively or negatively regulated to participate in the disease resistance-related responses of plants. In the experiment involving A. tenuissima infection, all treatments were carried out under natural light, and because A. tenuissima infection occurred during the day, sampling at 12 and $36 \mathrm{~h}$ after infection was conducted at night. The experiment revealed that PlWRKY65 expression in the blank control group was similar at 12 and $36 \mathrm{~h}$ but slightly higher at 24 and $48 \mathrm{~h}$ (Fig. 3). These results, together with the results for the G-box light-responsive components in the gene sequence, indicate that the expression of
PlWRKY65 may be induced by optical signals. At $48 \mathrm{~h}$ after infection, the outdoor temperature plummeted, accompanied by rain, which may have been the reason for the significant increase in expression in the blank control at 72 and $96 \mathrm{~h}$.

It has been found that plants infected by pathogens tend to accumulate plant protectors, such as phytoalexins, scopolin, and scopoletin around the invasion site, and the synthesis of these protectors depends on the JA, SA, and ethylene signaling pathways in plants ${ }^{29-31}$. However, most studies have revealed that WRKY TFs can mediate multiple disease resistance response pathways to regulate the expression of disease resistance-related genes, including the JA and SA signaling pathways ${ }^{6}$. In Catharanthus roseus, JA can promote the transcriptional accumulation of CrWRKY1, CrWRKY8, CrWRKY13, and CrWRKY $38^{32}$. Under MeJA treatment, GhWRKY4O gene expression is upregulated and positively regulates the resistance of cotton plants to Ralstonia solanacearum ${ }^{33}$. Rice OsWRKY13 is co-induced by SA and JA, and studies have surprisingly shown that OsWRKY13 in turn affects the accumulation of SA; that is, plants that overexpress OsWRKY13 accumulate more free $\mathrm{SA}^{8}$. In this study, PlWRKY65 silencing caused a decrease in JA content and an increase in SA content, which indirectly indicated that PlWRKY65 might promote JA accumulation and inhibit SA synthesis.

In the complex network through which plants regulate their response to external injury, there is an obvious antergic relationship between the SA and JA signaling 
pathways, and WRKY TFs mostly play a regulatory role at the junction of their signaling pathways ${ }^{34}$. Rice OsWRKY13, as a TF that directly or indirectly regulates disease resistance via the SA and JA signaling pathways, activates the transcription of SA synthesis-related genes and SA-induced genes and inhibits the expression of JA synthesis-related genes and JA-induced genes ${ }^{8,35}$. Erwinia carotovora infection in wild-type Arabidopsis can induce increased AtWRKY70 gene expression, which has been proven to be associated with elevated levels of endogenous SA. At the initial stage of infection, the transient increase in the JA level inhibits the expression of AtWRKY7O. Studies have concluded that AtWRKY7O is a transcriptional activator of SA-inducing genes and a transcriptional repressor of JA-inducing genes, leading to the intersection of the SA- and JA-mediated signal defense pathways ${ }^{36}$. Our research clearly showed that after infection with $A$. tenuissima, the endogenous JA content increased, but the content of endogenous SA decreased, and the two hormones showed opposite trends $96 \mathrm{~h}$ after infection. These results not only confirmed the mutual inhibition of the two hormones but also suggested that PlWRKY65 may be positively regulated in the JAmediated signaling pathway and may inhibit SA signaling pathway regulation. SA is an indispensable regulator that mainly combats infections involving biotrophic pathogens, such as Hyaloperonospora parasitica and Oidium neolycopersici ${ }^{37}$; however, JA-related defense mechanisms protect against necrotrophic pathogens such as Alternaria brassicicola and Botrytis cinerea ${ }^{38,39}$. As a necrotrophic pathogen, $A$. tenuissima is likely to cause the activation of JA signaling when it infects plants, which further explains the increase in JA content after infection with $A$. tenuissima.

The sudden onset of PTI and ETI in plants is usually regulated by hormone signaling pathways. The best studied of these pathways are those involving SA, JA, and ethylene, and these endogenous hormones can induce the expression of $P R$ genes ${ }^{40,41}$. In Arabidopsis, SA activates the expression of the AtPR-1,AtPR-2, and AtPR-5 genes, while JA activates the expression of the AtPR-3, AtPR-4, and AtPR-12 genes ${ }^{42}$. MeJA can induce the expression of MaPR5-2 and MaPR5-3, while both SA and MeJA can induce high MaPR1-1, MaPR2, and MaPR10c expression in banana fruit ${ }^{43}$. Signal transduction in the plant immune response is dependent on TFs. It has been reported that some pathogens and pathogen-derived elicitors, including SA and JA, can induce the expression of WRKY TFs, and some WRKY TFs are in turn involved in both the SA and JA pathways, showing the intersection of these effects ${ }^{5,44}$. Furthermore, WRKY TFs can regulate the expression of some $P R$ genes by binding to their promoters ${ }^{5}$. Rice OsWRKY3 can promote the expression of OsPR1 downstream as a transcriptional activator ${ }^{35}$. In rice, transgenic

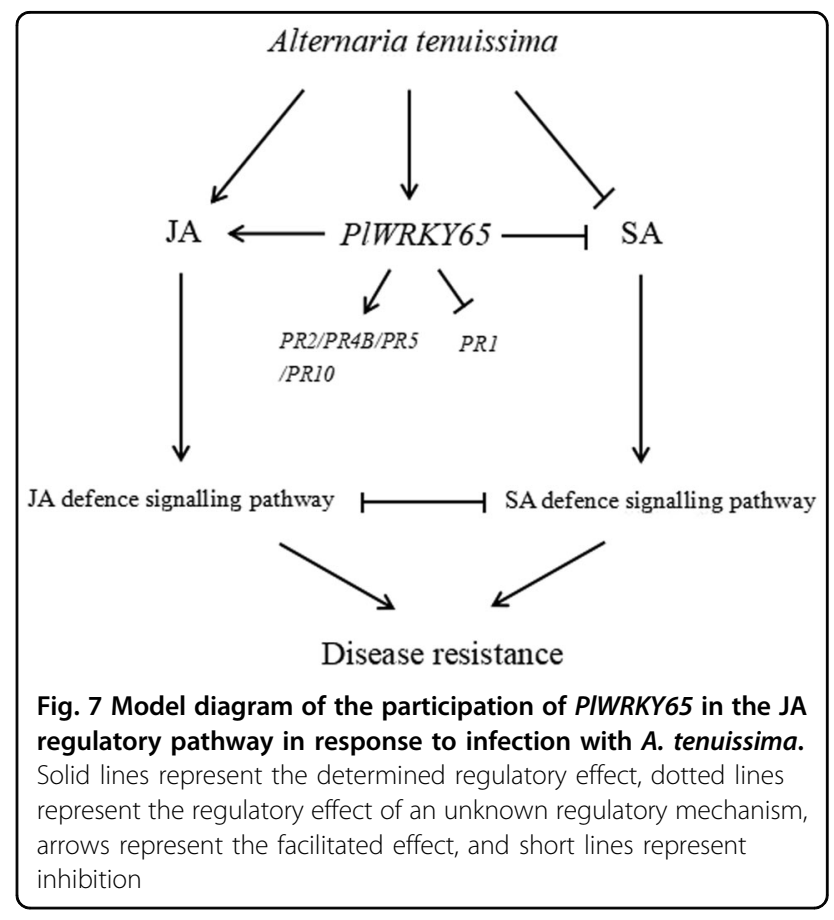

lines overexpressing OsWRKY28, OsWRKY71, OsWRKY76, and OsWRKY62 activate the pathogenesisrelated gene OsPR10, contributing to resistance against Xanthomonas oryzae pv. oryzae (Xoo) ${ }^{45}$. In our study, decreased PlWRKY65 expression resulted in significantly decreased PlPR2, PlPR4B, PlPR5, and PIPR10 expression and upregulated the transcriptional abundance of PlPR1. This illustrates that PlWRKY65 participates in the regulation of these PlPRs and may be involved in JAmediated and SA-mediated signaling pathways. An important finding is that PlPR1 is likely to be highly induced by SA and participate in the SA signaling pathway.

It can be concluded that PlWRKY65, acting as a transcriptional activator responding to pathogen induction, can mediate pathogen resistance by regulating PlPR gene expression, which may be partly due to SA-induced and JA-induced resistance. This study broadens our knowledge of the involvement of peony WRKY TFs in pathogen resistance (Fig. 7).

\section{Materials and methods}

\section{Plant materials and treatments}

P. lactiflora 'Da Fugui' was potted at the experimental forestry station of Shandong Agricultural University, Tai'an, Shandong, China, and plants were selected as experimental materials when they were in the middle stage of leaf development. The wound infection method was adopted for pathogen infection. Strain W23 of A. tenuissima preserved in our laboratory was cultured on solid PDA medium for 7 days, and the fungal colony edge 
was collected as the infection source and transferred to microwounds on leaves punctured with a sterilized insect needle $^{46}$. Leaves were collected at $0,12,24,36,48,72$, and $96 \mathrm{~h}$ after infection. Infection was observed and recorded in 20-30 healthy leaves showing consistent growth for each plant. The incidence grade for peony was calculated according to $\mathrm{Li}^{9}$, and the corresponding disease index was calculated as follows: disease index $=\sum$ (number of disease-grade plants $\times$ the representative value $\times 100 \% /$ plant number $x$ the representative value for the most severe disease. All samples were stored at $-80^{\circ} \mathrm{C}$ after quick freezing with liquid nitrogen. The control group was set up as described above, and each treatment was repeated three times.

Nicotiana benthamiana was cultured on an appropriate substrate and placed in a light incubator $\left(25^{\circ} \mathrm{C}, 120 \mathrm{~mol}\right.$ $\mathrm{m}^{-2} \mathrm{~s}^{-1}$, with a light/dark period of $16 \mathrm{~h} / 8 \mathrm{~h}$ ) for constant-temperature cultivation. When the plants had produced eight true leaves, the subcellular localization test was carried out.

\section{Total RNA extraction and CDNA synthesis}

The total RNA of the plant materials was extracted according to the instructions of the Aidlab EASYspin Rapid RNA Extraction Kit (Aidlab Biotech, Beijing, China). First-strand cDNA synthesis was performed by using the ComWin Biotech Reverse Transcription Kit (ComWin Biotech, Beijing, China).

\section{Cloning and sequence analysis of the PIWRKY65 gene}

The PlWRKY65 gene was screened according to the unigene functional annotation of the WRKY genes in the transcriptome data of $P$. lactiflora 'Da Fugui'; its openreading frame (ORF) sequence was predicted, and specific primers were designed (Table 1). The total DNA of $P$. lactiflora ' $\mathrm{Da}$ Fugui' was extracted via the improved CTAB method, and the full-length PlWRKY65 gene was cloned using total DNA as a template. The NCBI BLAST program (https://blast.ncbi.nlm.nih.gov/Blast.cgi) was used to screen out some amino acid sequences sharing high homology with P. lactiflora PIWRKY65, and DNAMAN 5.0 software was then used for multiple-sequence alignment to analyze the structure of the gene domains and conservation. MEGA 5.0 software was used to generate the evolutionary tree of the system for homology analysis.

\section{Subcellular localization}

The full-length cDNA of PlWRKY65 with the termination codon removed was used as a template, and specific primers with restriction sites (BamHI and KpnI) were designed for PCR amplification (Table 1). After enzyme digestion, the obtained product was ligated to the pROKII-GFP vector, which was also double digested, and
Table 1 Primers used in the tests and their sequences

\begin{tabular}{|c|c|c|}
\hline Primer name & Nucleotide sequence $\left(5^{\prime}-3^{\prime}\right)$ & Purpose \\
\hline PIActionF & ACTGCTGAACGGGAAATT & \multirow[t]{2}{*}{ Actin primers } \\
\hline PIActionR & ATGGCTGGAACAGGACTT & \\
\hline PIWRKY65aF & TTTGCCGAAGAGAGAAGCGT & \multirow{2}{*}{$\begin{array}{l}\text { Specific primer for } \\
\text { qRT-PCR }\end{array}$} \\
\hline PIWRKY65qR & TATACCTCCGTCGCTCCTCC & \\
\hline PIWRKY65F & ATGGACAGTCTATACAAT & \multirow{2}{*}{$\begin{array}{l}\text { Full-length DNA and } \\
\text { cDNA amplification }\end{array}$} \\
\hline PIWRKY65R & TCACGAGGTGGTCCCACAC & \\
\hline PIWRKY65(B)F & CGGGATCCATGGACAGTCTATAC & \multirow{2}{*}{$\begin{array}{l}\text { Vector construction } \\
\text { for GFP }\end{array}$} \\
\hline PIWRKY65(K)R & GGGGTACCCGAGGTGGTCCCAC & \\
\hline PIWRKY65(E)F & CGGAATTCAACCACCATGCCACC & \multirow{2}{*}{$\begin{array}{l}\text { Vector construction } \\
\text { for VIGS }\end{array}$} \\
\hline PIWRKY65(K)R & GGGGTACCCGAGGTGGTCCCACA & \\
\hline pTRV1F & TTACAGGTTATTTGGGCTAG & \multirow{4}{*}{$\begin{array}{l}\text { Molecular } \\
\text { detection of TRV }\end{array}$} \\
\hline pTRV1R & CCGGGTTCAATTCCTTATC & \\
\hline pTRV2F & TTTATGTTCAGGCGGTTCTTGTG & \\
\hline pTRV2R & CAAACGCCGATCTCAAACAGTC & \\
\hline PR1F & TACCCAGAGACGGTTCGACT & \multirow{10}{*}{$\begin{array}{l}\text { Primers for } \\
\text { pathogenesis- } \\
\text { related genes }\end{array}$} \\
\hline PR1R & CACACGAGTTGGACCGGTAA & \\
\hline PR2F & TGGCCAAAGGGGTCTCTAGA & \\
\hline PR2R & TCCCATTTACGGCAAGCGTA & \\
\hline PR4BF & ATCCCGCTCAACACTCTTGG & \\
\hline PR4BR & TCCACAGAAAGCAGTCCACC & \\
\hline PR5F & CAGTCTTCCCTCAGGCAAGG & \\
\hline PR5R & GGTTTCACATGCGGGTTTCC & \\
\hline PR10F & CCGGCAAGGATTITCAAGGC & \\
\hline PR10R & TTATCTTGATGGTCCCGGCG & \\
\hline
\end{tabular}

Note: The underlined 'GGATCC', 'GAATTC', and 'GGTACC' nucleotides are the added restriction enzyme recognition sites for BamHI, EcoRl, and Kpnl, respectively. Primers for the PTRV1 vector were designed within RNAdependent RNA polymerase elements (PCR product size of $647 \mathrm{bp}$ in theory), and primers for the PTRV2 vector were designed between MCS elements (PCR product size of $372 \mathrm{bp}$ in theory)

the fusion expression vector pROKII-PlWRKY65-GFP was verified by sequencing. Through Agrobacteriummediated infection, the recombinant vector and the empty vector without PlWRKY65 were introduced into tobacco leaves. After cultivation for 3 days, fluorescent sites were observed under a confocal laser scanning microscope (Nikon, Tokyo, Japan).

\section{VIGS in P. lactiflora}

To specifically silence the PlWRKY65 gene, we amplified a 402-bp fragment of the gene and cloned it into the pTRV2 vector. The correct recombinant vector was verified by PCR and sequencing.

The pTRV1, pTRV2, and pTRV2-WRKY65 plasmids were transformed into Agrobacterium tumefaciens GV3101. 
A $1 \mathrm{~mL}$ aliquot of $A$. tumefaciens GV3101-pTRV1, GV3101-pTRV2, and GV3101-pTRV2-WRKY65 was cultured in $10 \mathrm{~mL}$ of YEP liquid medium (including $50 \mu \mathrm{g} / \mathrm{mL}$ Kan and $100 \mu \mathrm{g} / \mathrm{mL}$ Rif) at $28^{\circ} \mathrm{C}$ for $24 \mathrm{~h}$ at $200 \mathrm{r} \mathrm{min}{ }^{-1}$. Then, $10 \mathrm{~mL}$ of the bacterial liquid was transferred to $400 \mathrm{~mL}$ of YEP liquid medium (including $50 \mu \mathrm{g} / \mathrm{mL}$ Kan, $100 \mu \mathrm{g} / \mathrm{mL}$ Rif, and $200 \mu \mathrm{M}$ acetosyringone (AS)) followed by culture at $28^{\circ} \mathrm{C}$ for $5-6$ days at $200 \mathrm{r} \mathrm{min}^{-1}$. When the $\mathrm{OD}_{600}$ of the bacterial liquid was $\sim 1.5$, the cells were centrifuged at $4{ }^{\circ} \mathrm{C}$ at $12,000 \mathrm{r} / \mathrm{min}$ for $2 \mathrm{~min}$, collected, and resuspended $\left(10 \mathrm{mmol} / \mathrm{L} \mathrm{MES,} 10 \mathrm{mmol} / \mathrm{L} \mathrm{MgCl}_{2}, 150 \mu \mathrm{M}\right.$ AS, and aseptic water as solvent). The $\mathrm{OD}_{600}$ of the suspension was adjusted to 1.5 . GV3101-pTRV2 and GV3101-pTRV2-WRKY65 were mixed with GV3101pTRV1 at a 1:1 ratio, and the mixture was allowed to rest for 3-5 $\mathrm{h}$ at room temperature in darkness ${ }^{47}$.

Peonies with strong and consistent growth were selected, and VIGS was carried out via the negative-pressure vacuum filtration method when the underground buds had broken through the soil and were about to produce leaves. The plants were carefully dug out of the pot and placed in a vacuum bucket containing the infection solution described above, ensuring that the plant was completely immersed in the infection solution and that the root system was not destroyed. The treated plants were returned the pots and bagged, then kept in the dark for $24 \mathrm{~h}$. Thereafter, the treated plants and control plants were subjected to normal field management procedures.

\section{Quantitative real-time PCR (qRT-PCR)}

In this experiment, qRT-PCR was used to determine gene expression. The instrument was a Bio-Rad CFX96 ${ }^{\mathrm{TM}}$ real-time system (Bio-Rad, Hercules, CA, USA), and the qRT-PCR mixture (total volume of $20 \mu \mathrm{L}$ ) contained $10 \mu \mathrm{L}$ of $\mathrm{SYBR}^{\circledast}$ Premix Ex Taq ${ }^{\mathrm{TM}}$ (TaKaRa, Inc., Japan), $8 \mu \mathrm{L}$ of $\mathrm{ddH}_{2} \mathrm{O}, 0.5 \mu \mathrm{L}$ of each primer, and $1 \mu \mathrm{L}$ of cDNA. The reaction procedure was as follows: $95^{\circ} \mathrm{C}$ for $30 \mathrm{~s} ; 40$ cycles of $95^{\circ} \mathrm{C}$ for $5 \mathrm{~s}$ and $60^{\circ} \mathrm{C}$ for $30 \mathrm{~s}$; and a dissociation stage of $95^{\circ} \mathrm{C}$ for $10 \mathrm{~s}, 65^{\circ} \mathrm{C}$ for $5 \mathrm{~s}$, and $95^{\circ} \mathrm{C}$ for $5 \mathrm{~s}$. For each test sample, three biological repeats were performed, and the data were analyzed using the $2^{-\Delta \Delta C T}$ method $^{48}$. The PlActin gene was used as the housekeeping gene to detect the expression levels of other genes.

\section{Determination of endogenous hormones}

The leaves of P. lactiflora were sampled at $0,12,24,48$, 72 , and $96 \mathrm{~h}$ after pathogen infection, and the levels of the endogenous hormones JA and SA were determined by high-performance liquid chromatography ${ }^{49}$. The chromatographic conditions were as follows: for JA, a RIGOL L3000 high-performance liquid chromatography instrument (RIGOL, Suzhou, China) was used with a wavelength of $210 \mathrm{~nm}$ and a Kromasil C18 reversed-phase chromatographic column $(250 \mathrm{~mm} \times 4.6 \mathrm{~mm}, 5$ micron $)$; the flow rate was $0.8 \mathrm{~mL} / \mathrm{min}$, the mobile phase was $1 \%$ phosphoric acid (aqueous solution):acetonitrile $=45: 55$ $(\mathrm{V} / \mathrm{V})$, and the sample volume was $10 \mu \mathrm{L}$. For SA, a Waters 1525 high-performance liquid chromatography instrument (Waters, Shanghai, China) was used with the fluorescence detector set at an excitation wavelength of $294 \mathrm{~nm}$ and emission wavelength of $426 \mathrm{~nm}$ and a Kromasil C18 reversed-phase chromatographic column ( $250 \mathrm{~mm} * 4.6 \mathrm{~mm}, 5$ micron); the flow rate was $0.8 \mathrm{~mL} /$ min; the mobile phase was $1 \%$ acetic acid solution: methanol $=2: 3(\mathrm{~V} / \mathrm{V})$; and the sample volume was $10 \mu \mathrm{L}$. Three biological repeats were performed for each sample.

\section{Statistical analysis}

At least three biological replicates were included in the data, and all data were analyzed using ANOVA and Student's $t$-test for the determination of significant differences by using SPSS 24.0 software.

\section{Acknowledgements}

This study was funded by the Shandong Provincial Agricultural Elite Varieties Project (2019 LZGC018), China.

\section{Author details}

${ }^{1}$ College of Forestry, Shandong Agricultural University, No. 61, Daizong Road, 271018 Tai'an, Shandong, China. ${ }^{2}$ Shandong Provincial Research Center of Demonstration Engineering Technology for Urban and Rural Landscapes, 271018 Tai'an, Shandong, China. ${ }^{3}$ Shandong Institute of Pomology, 271000 Tai'an, Shandong, China

Conflict of interest

The authors declare that they have no conflict of interest.

Received: 30 July 2019 Revised: 3 February 2020 Accepted: 7 February 2020 Published online: 01 April 2020

\section{References}

1. Peyraud, R. et al. Advances on plant-pathogen interactions from molecular toward systems biology perspectives. Plant J. 90, 720-737 (2017).

2. Yang, B., Jiang, Y. Q., Rahman, M. H., Deyholos, M. K. \& Kav, N. N. Identification and expression analysis of WRKY transcription factor genes in canola (Brassica napus $L$.) in response to fungal pathogens and hormone treatments. BMC Plant Biol. 9, 68 (2009).

3. Eulgem, T., Rushton, P. J., Robatzek, S. \& Somssich, I. E. The WRKY superfamily of plant transcription factors. Trends Plant Sci. 5, 199-206 (2000).

4. Rushton, D. L. et al. WRKY transcription factors: key components in abscisic acid signallingPlant Biotechnol. J. 10, 2-11 (2012).

5. Rushton, P. J., Somssich, I. E., Ringler, P. \& Shen, Q. J. WRKY transcription factors. Trends Plant Sci. 15, 247-258 (2010).

6. Tao, Z. et al. A pair of allelic WRKY genes play opposite roles in rice-bacteria interactions. Plant Physiol. 151, 936-948 (2009).

7. Li, C. et al. Cotton WRKY1 mediates the plant defense-to-development transition during infection of cotton by Verticillium dahliae by activating JASMONATE ZIM-DOMAIN1 expression. Plant Physiol. 166, 2179-2194 (2014).

8. Qiu, D. Y. et al. OsWRKY13 mediates rice disease resistance by regulating defense-related genes in salicylate- and jasmonate-dependent signaling. Mol. Plant Microbe Interact. 20, 492-499 (2007).

9. Li, L., Song, S. X., Liu, H. X. \& Guo, X. F. Identification of red spot pathogens on peony in Shandong province. Acta Hortic. Sin. 43, 365-372 (2016).

10. Li, J. B., Luan, Y. S. \& Liu, Z. Overexpression of SpWRKY1 promotes resistance to Phytophthora nicotianae and tolerance to salt and drought stress in transgenic tobacco. Physiol. Plant 155, 248-266 (2015). 
11. Cui, J. et al. Transcriptome signatures of tomato leaf induced by Phytophthora infestans and functional identification of transcription factor SpWRKY3. Theor. Appl. Genet. 131, 787-800 (2018).

12. Hong, Y. H., Cui, J., Liu, Z. \& Luan, Y. S. SpWRKY6 acts as a positive regulator during tomato resistance to Phytophthora infestans infection. Biochem. Biophys. Res. Commun. 506, 787-792 (2018).

13. Kim, K. C., Fan, B. F. \& Chen, Z. X. Pathogen-induced Arabidopsis WRKY7 is a transcriptional repressor and enhances plant susceptibility to Pseudomonas syringae. Plant Physiol. 142, 1180-1192 (2006).

14. Birkenbihl, R. P., Diezel, C. \& Somssich, I. E. Arabidopsis WRKY33 is a key transcriptional regulator of hormonal and metabolic responses toward Botrytis cinerea infection. Plant Physiol. 159, 266-285 (2012).

15. Hsu, F. C. et al. Submergence confers immunity mediated by the WRKY22 transcription factor in Arabidopsis. Plant Cell. 25, 2699-2713 (2013).

16. Li, J., Zhong, R. S. \& Palva, E. T. WRKY70 and its homolog WRKY54 negatively modulate the cell wall-associated defenses to necrotrophic pathogens in Arabidopsis. PLoS ONE 12, e0183731 (2017).

17. Hara, K., Yagi, M., Kusano, T. \& Sano, H. Rapid systemic accumulation of transcripts encoding a tobacco WRKY transcription factor on wounding. Mol. Gen. Genet. 263, 30-37 (2000).

18. Kim, C. Y. et al. Identification of rice blast fungal elicitor-responsive genes by differential display analysis. Mol. Plant-Microbe Interact. 13, 470-474 (2000).

19. Cormack, R. S. et al. Leucine zipper-containing WRKY proteins widen the spectrum of immediate early elicitor-induced WRKY transcription factors in parsley. Biochim. Biophys. Sci. Acta 1576, 92-100 (2002).

20. Sun, C. et al. A novel WRKY transcription factor, SUSIBA2, participates in sugar signaling in barley by binding to the sugarresponsive elements of the isol promoter. Plant Cell 15, 2076-2092 (2003).

21. Xu, Y. H., Wang, J. W., Wang, S., Wang, J. Y. \& Chen, X. Y. Characterization of GaWRKYl, a cotton transcription factorthat regulates the sesquiterpene synthase gene (+)-delta-cadinene synthase-A. Plant Physiol. 135, 507-515 (2004).

22. Singh, V., Roy, S., Singh, D. \& Nandi, A. K. Arabidopsis FLOWERING LOCUS D influences systemic-acquired-resistance-induced expression and histone modifications of WRKY genes. J. Biosci. 39, 119-126 (2014).

23. Luo, X. et al. Ectopic expression of a WRKY homolog from Glycine soja alters flowering time in Arabidopsis. PLOS ONE 8, e73295 (2013).

24. Shi, W. et al. The Gossypium hirsutum WRKY gene GhWRKY39-1 promotes pathogen infection defense responses and mediates salt stress tolerance in transgenic Nicotiana benthamiana. Plant Cell Rep. 33, 483-498 (2014).

25. Wang, L. et al. Genome-wide identification of WRKY family genes and their response to cold stress in Vitis vinifera. BMC plant Biol. 14, 103 (2014).

26. Chen, L., Zhang, Y. \& Yu, D. Wounding-induced WRKY 8 is involved in basal defense in Arabidopsis. Mol. Plant Microbe Interact. 23, 558-565 (2010).

27. $\mathrm{Wu}, \mathrm{L}$. T. et al. Arabidopsis WRKY28 transcription factor is required for resistance to necrotrophic pathogen, Botrytis cinerea. Afr. J. Microbiol. Res. 5, 5481-5488 (2011).

28. Atamian, H. S., Eulgem, T. \& Kaloshian, I. SIWRKY70 is required for Mi-1mediated resistance to aphids and nematodes in tomato. Planta 235 299-309 (2012).

29. Sun, $\mathrm{H}$. et al. Scopoletin is a phytoalexin against Alternaria alternata in wild tobacco dependent on jasmonate signalling. J. Exp. Bot. 65, 4305-4315 (2014).

30. Li, J. \& Wu, J. Scopolin, a glycoside form of the phytoalexin scopoletin, is likely involved in the resistance of Nicotiana attenuata against Alternaria alternata. J. Plant Pathol. 98, 641-644 (2016).
31. Sun, $H$. et al. Ethylene signalling is essential for the resistance of Nicotiana attenuata against Alternaria alternata and phytoalexin scopoletin biosynthesis. Plant Pathol. 66, 277-284 (2017).

32. Craig, S., Sitakanta, P., Barunava, P. \& Ling, Y. Analyses of Catharanthus roseus and Arabidopsis thaliana WRKY transcription factors reveal involvement in jasmonate signaling. BMC Genomics 15, 502 (2014).

33. Wang, X. et al. GhWRKY40, a multiple stress-responsive cotton WRKY gene, plays an important role in the wounding response and enhances susceptibility to Ralstonia solanacearum infection in transgenic Nicotiana benthamiana. PLOS ONE 9, e93577 (2014).

34. Kunkel, B. N. \& Brooks, D. M. Cross talk between signaling pathways in pathogen defense. Curr. Opin. Plant Biol. 5, 325-331 (2002).

35. Liu, X. Q. et al. OsWRKY03, a rice transcriptional activator that functions in defense signaling pathway upstream of OsNPR1. Cell Res. 15, 593-603 (2005).

36. Li, J., Brader, G. \& Palva, E. T. The WRKY70 transcription factor: a node of convergence for jasmonate-mediated and salicylate-mediated signals in plant defense. Plant Cell 16, 319-331 (2004).

37. Mammarella, N. D. et al. Apoplastic peroxidases are required for salicylic acidmediated defense against Pseudomonas syringae. Phytochemistry 112, 110-121 (2015).

38. De, C. B., Timmermans, P., Vos, C., Cammue, B. P. A. \& Kazan, K. What lies beneath: belowground defense strategies in plants. Trends Plant Sci. 20, 91-101 (2015).

39. Adie, B. A. et al. ABA is an essential signal for plant resistance to pathogens affecting JA biosynthesis and the activation of defenses in Arabidopsis. Plant Cell. 19, 1665-1681 (2007).

40. Van Loon, L. C., Rep, M. \& Pieterse, C. M. J. Signifificance of inducible defenserelated proteins in infected plants. Annu. Rev. Phytopathol. 44, 135-162 (2006).

41. Robert-Seilaniantz, A., Grant, M. \& Jones, J. D. G. Hormone crosstalk in plant disease and defense: more than just jasmonate-salicylate antagonism. Annu. Rev. Phytopathol. 49, 317-343 (2011).

42. Thomma, B. P. et al. Separate jasmonate-dependent and salicylatedependent defense-response pathways in Arabidopsis are essential for resistance to distinct microbial pathogens. Proc. Natl Acad. Sci. USA 95 15107-15111 (1998).

43. Yang, $T$. et al. Molecular characterization of PR and WRKY genes during SAand MeJA-induced resistance against Colletotrichum musae in banana fruit. Postharvest Biol. Technol. 79, 62-68 (2013).

44. Mao, P., Duan, M. R., Wei, C. H. \& Li, Y. WRKY62 transcription factor acts downstream of cytosolic NPR1 and negatively regulates jasmonate-responsive gene expression. Plant Cell Physiol. 48, 833-842 (2007).

45. Peng, Y., Bartley, L. E., Canlas, P. \& Ronald, P. C. OsWRKY lla transcription factors modulate rice innate immunity. Rice 3, 36-42 (2010).

46. Tan, G. J., Li, Z. Z., Liu, S. F., Wang, G. \& Huang, Y. K. Mechanisms of action and efficacy of Bacillus subtilis BS80-6 active against postharvest anthracnose pathogen on apples. Acta Phytophylacica Sin. 35, 227-232 (2008).

47. Bachan, S. \& Dinesh-Kumar, S. P. Tobacco rattle virus (TRV) based virus-induced gene silencing. Methods Mol. Biol. 894, 83-92 (2012).

48. Schmittgen, T. D. \& Livak, K. J. Analyzing real-time PCR data by the comparative CT method. Nat. Protoc. 3, 1101-1108 (2008).

49. Dewdney, J. et al. Three unique mutants of Arabidopsis identify eds loc required for limiting growth of a biotrophic fungal pathogen. Plant J. 24, 205-218 (2000) 